Journal of Case Reports 2021;11(3):156-159

\title{
Traumatic Pseudoaneurysm of the Radial Artery
}

\author{
K Aghedo, EW Ugboma, OD Ray-Offor, VO Madukaife \\ Department of Radiology, University of Port Harcourt Teaching Hospital, Alakahia Rivers State, Nigeria.
}

\author{
Corresponding Author: \\ Dr K Aghedo \\ Email: draghedokawa@gmail.com \\ This is an Open Access article distributed \\ under the terms of the Creative Commons \\ Attribution License (creativecommons.org/ \\ licenses/by/3.0). \\ Received : December 1, 2020 \\ Accepted : April 26, 2021 \\ Published : July 25, 2021
}

\begin{abstract}
Background: Pseudoaneurysm patients usually present months later following arterial injury with progressive swelling and pain at the initial site of injury. Quick intervention is needed to prevent complications such as thromboembolism, infection, rupture, neuropraxia and compartment syndrome. Case Report: A rare pseudoaneurysm of radial artery is being reported which was initially missed. A duplex scan confirmed the diagnosis with the patient undergoing a surgical repair of the vessel. Conclusion: Pseudoaneurysm of the forearm arteries is uncommon and ultrasound plays an important role in distinguishing vascular pathologies from other masses.
\end{abstract}

Keywords: False Aneurysm, Forearm, Pain, Radial Artery, Thromboembolism.

\section{Introduction}

Pseudoaneurysm is a rare and late complication of an arterial lesion [1]. These lesions are missed at the time of the initial injury and hence patients present months later with progressive swelling and pain at the initial site of injury. Quick intervention is required to prevent complications such as thromboembolism, infection, rupture, neuropraxia and compartment syndrome [2].

Pseudoaneurysm of the radial artery can follow trauma, iatrogenic vascular interventions, anastomotic disruption, and in intravenous drug abusers [3]. The incidence of pseudoaneurysm has increased in hospital-based practice as a result of the large number of invasive procedures performed [4]. The reported incidence of radial artery pseudoaneurysm is $0.05 \%$ [5]. The radial artery in its distal part is more superficial and hence susceptible to trauma. The need for early recognition and referral for surgical management cannot be overemphasized to prevent these complications. Early imaging (ultrasonography) helps in differentiating this vascular lesion from other pathologies [1]. This case is presented because of the rarity of the pathology and to emphasize the usefulness of ultrasound as an important tool for confirmation of clinical diagnosis.

\section{Case Report}

N.H is a 60-year-old female, a farmer who reported to the hospital with 9-month history of swelling on the volar aspect of the right distal forearm. She had a knife cut at the site of the swelling, year prior to presentation for which she was treated by a chemist who dressed the wound and subsequently it healed. The swelling was painful, had progressively increased in size and she felt pulsating sensation at the site of the swelling. No history of change in colour of the hand, fever, numbness, or paraesthesia was noted.

Physical examination revealed an elderly woman who was not pale, afebrile, and anicteric. Musculoskeletal examination showed a round swelling about $4 \mathrm{~cm}$ in its widest diameter with a scar on the distal half of the right forearm $4 \mathrm{~cm}$ from the radial styloid process. The swelling was firm, pulsatile, not attached to the overlying skin 
or underlying structures. It was not fluctuant or compressible, did not transilluminate and the radial pulse was felt distal to the swelling. Proximal to the swelling there was difficulty in accessing the radial artery due to associated tenderness. Allen's test was however negative indicating a reduced risk of ischemia to the palm. Doppler ultrasound scan of the swelling and plain radiograph of the right forearm was requested. Plain radiograph of the right forearm showed a round soft tissue opacity at the lateral aspect of the distal forearm. No calcification, lucency or radiopaque foreign body was seen within the opacity. The bones were normal [Fig.1,2]. Duplex scan revealed a partially thrombosed pseudoaneurysm in the distal radial artery with evidence of to and fro flow. It measured $1.76 \times 2.48 \mathrm{~cm}$. There was damping of flow distal to the aneurysm. Low resistance flow was noted, and the velocity range was $50-80 \mathrm{~cm} / \mathrm{s}$ [Fig.3-5]. A diagnosis of pseudoaneurysm of the distal radial artery was made. Patient was admitted into the surgical ward and billed for surgical repair of right radial artery aneurysm. Surgical findings included ventral aneurysm of the right radial artery proximal to the wrist joint. The resected aneurysmal tissue was sent for histology. Histology report confirmed the pseudoaneurysm of the right distal radial artery. At one year follow up, patient was asymptomatic with no post-surgical complications.

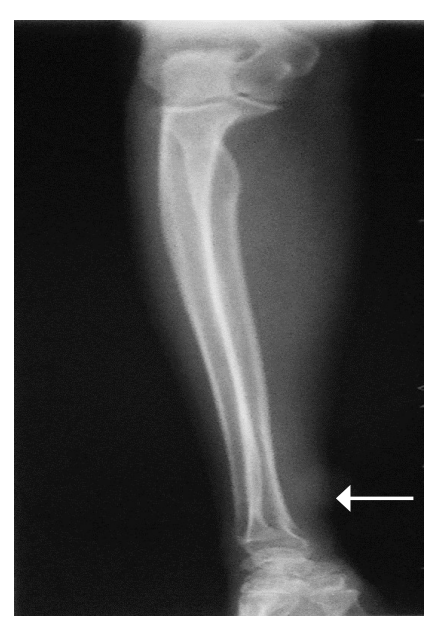

Fig.1: Anteroposterior radiograph of the right forearm showing a soft tissue density at the distal aspect of the forearm (white arrow).

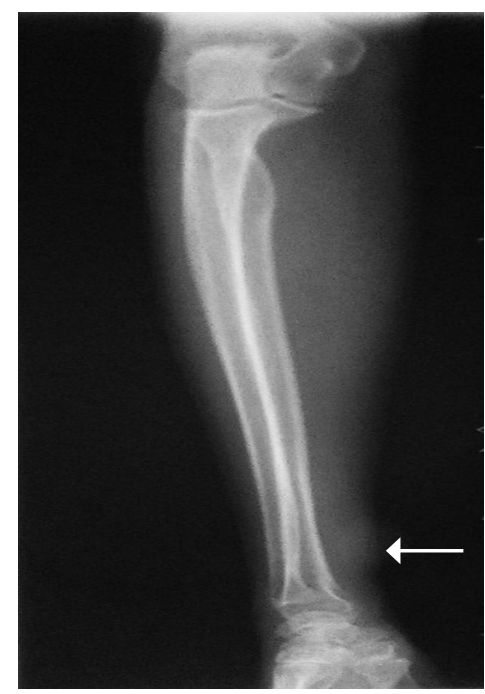

Fig.2: Lateral radiograph of the right forearm showing a soft tissue density in the posterior aspect of the distal forearm (white arrow).

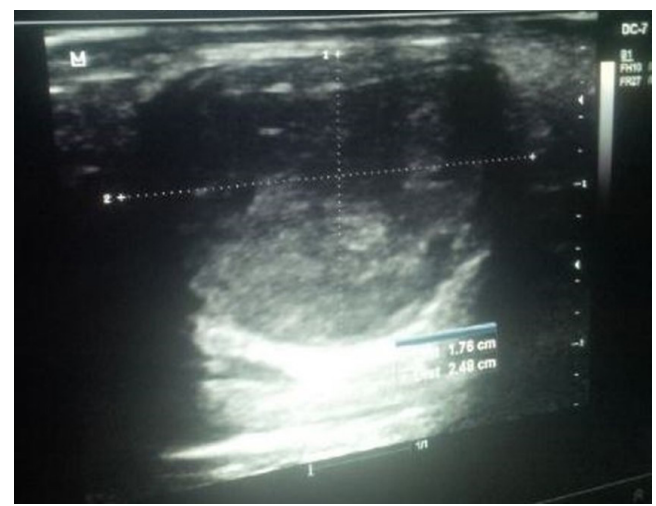

Fig.3: Gray scale ultrasound image showing a partially thrombosed pseudoaneurysm measuring $1.76 \times 2.48 \mathrm{~cm}$.

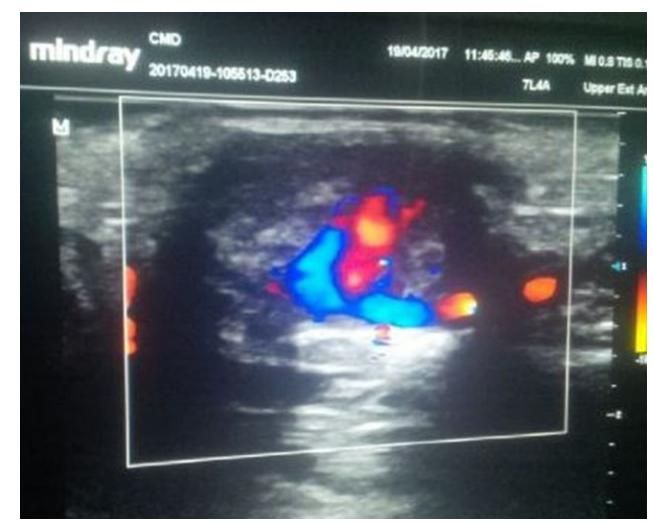

Fig.4: Duplex ultrasound image showing an aneurysmal sac and turbulent flow ("yin-yang" sign) within the sac. 


\section{Discussion}

Pseudoaneurysm is also known as false aneurysm and it occurs when there is a breach in the vessel wall [2]. This results in leakage of blood through the wall, which is contained by the adventitia or surrounding perivascular soft tissue. Hence a direct communication of blood flow exists between the vessel lumen and the aneurysm lumen through the hole in the vessel wall. A false aneurysm does not have the three layers of the arterial wall whereas a true aneurysm has all the three layers of the wall (intima, media and adventitia) [2]. The frequency of pseudoaneurysms in the upper extremities is much lower than that in the lower extremities [3]. The incidence of pseudoaneurysms has increased in hospital-based practice as a result of the large number of invasive procedures performed [4]. The radial artery is commonly affected on its distal segment where it is more superficial as seen in the index case.

Pseudoaneurysm of the radial artery can occur as a result of trauma (penetrating or blunt), iatrogenic vascular interventions, anastomotic disruption, and in intravenous drug abusers [5]. The index patient had a penetrating injury as the causative factor. This vascular lesion (pseudoaneurysm) can occur within hours or months from the time of injury as in the case under review which occurred months after the initial injury [6]. False aneurysms due to penetrating or blunt trauma may be seen in patients of every age and at any location. The pseudoaneurysms of the distal upper extremity may present as many other soft tissue masses and as such diagnostic puncture of a wrist swelling may be misguided [6]. Patients may present with a painful palpable pulsatile or non-pulsatile expanding mass with or without compromising the distal limb [7]. The index patient presented with a palpable pulsating mass without compromising the distal limb. On physical examination, Allen's test is usually negative and arterial pulses are usually felt distal to the mass in pseudoaneurysm. The goal of imaging

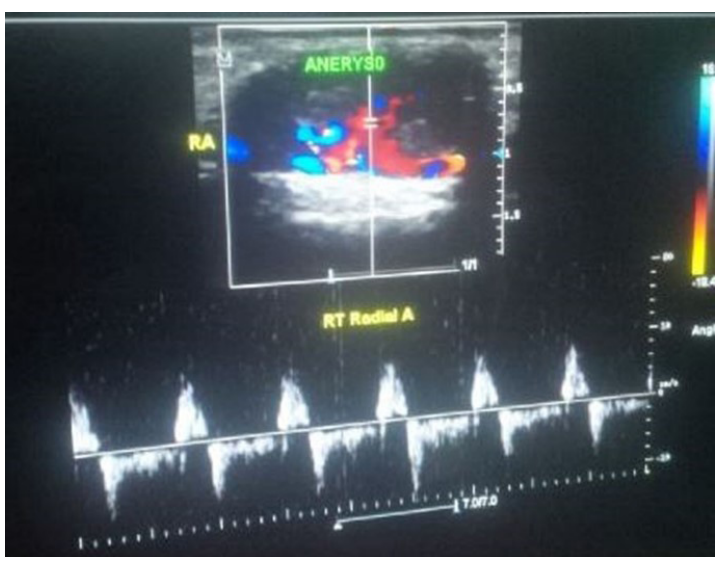

Fig.5: Duplex ultrasound image demonstrating the to-andfro (bidirectional) flow.

is to differentiate between hematomas associated with pseudoaneurysm and those that are not. Ultrasonography (US) is the preferred imaging modality to differentiate pseudoaneurysm from other masses [8]. This is because it is readily available, fast, portable, non-invasive, less expensive, does not involve the use of ionising radiation and renal toxic contrasts. Other imaging modalities that could be used to diagnose pseudoaneurysm include computed tomography (CT) angiography, magnetic resonance angiography and conventional angiography [2]. Contrast enhanced computed tomography may show a contrast material filled sac with a hypodense area indicating thrombosis [8]. Three-dimensional CT angiography and gadolinium-enhanced magnetic resonance (MR) angiography can show the lesion in any projection and from various angles. CT angiography is however limited by imaging artifacts from bullets fragments or other metallic objects. The use of ionizing radiation and iodinated contrast materials are also drawbacks. MR angiography is time consuming when compared with US or CT, has limited availability and proper patient monitoring is difficult [8]. Conventional angiography has the ability to assess real time hemodynamic of a specific vascular bed. It is however invasive, cannot assess the size of a pseudoaneurysm that contains a thrombus and has an increased risk of procedure related 
complications such as hematomas, arteriovenous fistula, ischemia etc [2]. On duplex ultrasonography, the main appearances of pseudoaneurysm are presence of a hematoma with variable echogenicity which indicates different episodes of bleeding and re-bleeding, an expansile pulsatility and detection of turbulent flow which is seen as the classic "yinyang" sign. The "to-and-fro" spectral waveform pattern when seen within the neck connecting the pseudoaneurysm to the injured radial artery is pathognomonic [9]. These findings were noted in the index case.

Small pseudoaneurysm may likely thrombose spontaneously over time however the morbidity associated with pseudoaneurysm may be severe [9]. This is because of distal embolization from micro-emboli, rupture, infection, and venous compression. The management of pseudoaneurysm is dependent on the cause, site, presence of thrombi, associated symptoms, and circulation status collaterally and distally to the lesion [7]. Various treatment options for radial artery pseudoaneurysm include observation, compression bandages, ultrasound guided compression, ultrasound guided thrombin injection and surgical repair. Indications for surgical repair include symptomatic expanding infection, prolonged history with large hematomas and failed conservative treatment [9]. In this index case, surgical option was chosen based on the duplex ultrasound findings as patient did not show signs of ischemia.

\section{Conclusion}

Pseudoaneurysm of the forearm arteries is uncommon and ultrasound plays an important role in distinguishing vascular pathologies from other masses.

Contributors: KA: manuscript writing, patient management; EWU: manuscript editing, patient management; ODRO, VOM: critical inputs into the manuscript. KA will act as a study guarantor. All authors approved the final version of this manuscript and are responsible for all aspects of this study.

Funding: None; Competing interests: None stated.

\section{References}

1. Pero T, Herrick J. Pseudoaneurysm of the radial artery diagnosed by bedside ultrasound. West J Emerg Med. 2009;10(2):89.

2. Saad NEA, Saad WEA, Davies MG, Waldman DL, Fultz PJ, Rubens DJ. Pseudoaneurysms and the role of minimally invasive techniques in their management. RadioGraphics. 2005;25(suppl_1):S173-S89.

3. Nwafor I, Eze J, Ezemba N, Onyekwulu F, Ngene C, Chinawa J. The pattern and outcome of civilian vascular injury in a teaching hospital over a 7-year period. WJCD. 2016;6(02):44-46.

4. Fransson SG, Nylander E. Vascular injury following cardiac catheterization, coronary angiography, and coronary angioplasty. Eur Heart J. 1994;15:232-235.

5. Cozzi DA, Morini F, Casati A, Pacilli M, Salvini V, Cozzi F. Radial artery pseudoaneurysm successfully treated by compression bandage. Arch Dis Child. 2003;88:165166.

6. Leone V, Misuri D, Console N. Radial artery pseudoaneurysm after a single arterial puncture for blood-gas analysis: a case report. Cases Journal. 2009;2(1):6890.

7. Patel K, Gandhi S, Sutariya H. Radial artery pseudoaneurysm: A rare complication after a single arterial puncture for blood-gas analysis. Indian J Crit Care Med. 2016;20(10):622-626.

8. Kapoor BS, Haddad HL, Saddekni S, Lockhart ME. Diagnosis and management of pseudoaneurysms: an update. Curr Probl Diagn Radiol. 2009;38:170-188.

9. Rozen G, Samuels D, Blank A. The to and fro sign: the hallmark of pseudoaneurysms. Isr Med Assoc J. 2001; 3:781-782. 